\title{
INFANTILE MYOFIBROMATOSIS- A CASE REPORT
}

M. Ramani, R. Sowjanya, O. H. Radhika, K. Ramesh Reddy.

1. Professor, Department of Pathology, Niloufer Hospital for Women and Children.

2. Post graduate, Department of Pathology, Niloufer Hospital for Women and Children.

3. Assistant professor, Department of Pathology. Niloufer Hospital for Women and Children.

4. Professor and HOD, Department of Paediatric surgery, Niloufer Hospital for Women and Children.

\section{CORRESPONDING AUTHOR:}

Dr Ramani M.

Professor Department of Pathology,

Osmania Medical College,

Koti, Hyderabad,

Andhra Pradesh, India.

E-mail: drmramani@sify.com

ABSTRACT: Infantile myofibromatosis is a rare, benign proliferative myofibroblastic tumour which occurs mostly in infants and young children. It may present as a solitary lesion most commonly involving skin, bone, muscle, subcutaneous tissue, in head, neck and trunk, with good prognosis, or a multicentric form of infantile myofibromatosis with or without visceral involvement (heart, lung, gastrointestinal tract, and kidney) with a poor prognosis. We report a case of one month old male child who presented with a subcutaneous nodule over the trunk. The mass was excised and histopathologically, immunohistochemically diagnosed as infantile myofibromatosis.

KEYWORDS: Infantile myofibromatosis, newborn, trunk.

INTRODUCTION: Infantile myofibromatosis is a rare mesenchymal neoplasm of infancy and early childhood, typically presenting as single or multicentric nodular masses of soft tissues, bones, or visceral organs. First described by Stout in 19543, various terms such as congenital multiple fibromatosis, multiple mesenchymal hamartomas, and diffuse congenital fibromatosis have been used to describe the same entity. Chung and Enzinger ${ }^{4}$ used the term "infantile myofibromatosis" for the first time after reviewing 61 cases. There are three different clinical entities of infantile myofibromatosis, including (1) solitary form characterized by a single nodular lesion, (2) multicentric form without apparent visceral involvement, and (3) multicentric form with visceral involvement. Patients with visceral involvement have been reported to have poor prognosis. Indolent clinical course and spontaneous tumor regression may occur in the two forms of infantile myofibromatosis without visceral involvement. Finally, in 1989, Smith et al. and Daimaru et al. coined the terms "myofibromas" and "myofibromatosis." This nomenclature was adopted by the World Health Organization (WHO) to describe the solitary form (myofibromas) or multicentric form (myofibromatosis) ${ }^{1}$.

CASE REPORT: A one month old male child presented with a swelling over the lower back on left side. The swelling was present since birth with gradual increase in size. There were no other complaints. Family history was unremarkable. Palpation revealed a subcutaneous swelling 
measuring $4 \times 4 \mathrm{~cm}$, firm in consistency. Ultrasonography showed well circumscribed cystic lesion with echogenic content, suggestive of abscess. Excision biopsy was done and specimen sent for histopathology. Post operative period was nil remarkable.

GROSS - We received a globular soft tissue mass measuring $4 \times 4 \times 2.5 \mathrm{~cm}$. Cut section was solid, homogeneous grey-white.

MICROSCOPY - Multiple sections studied from the mass showed interlacing fascicles of spindle cells in a collagenised background alternating with hypocellular areas. Smooth muscle, hemangiopericytoma like areas and fibroblasts were also seen. Immunohistochemistry was performed and was positive for Vimentin. Features were suggestive of infantile myofibromatosis solitary form.

DISCUSSION: Infantile myofibromatosis is a rare mesenchymal tumour usually found in the first decade of life with $88 \%$ of cases detected before the age of 2 years and $60 \%$ at or shortly after birth. Lesions have been found in nearly all kinds of tissues, including the bone, lip, oral cavity, central nervous system, gastrointestinal tract, lungs, myocardium, liver, and biliary tree ${ }^{4-7}$. The most common clinical manifestation is the presence of discrete nodules in the skin, muscle, or subcutaneous tissues.

Study of 199 cases of infantile myofibromatosis by Jenkins and Cawley ${ }^{11}$ revealed that the multicentric form (56\%) was more common than the solitary form (44\%). The solitary form occurred predominantly in boys (61\%) primarily in soft tissue of the head, neck and trunk, so was in our case.

Superficial skin lesions may resemble hemangiomas due to prominent tumor vascularity. In multicentric form, the number of lesions varies from 2 to 100, and they may be mistaken for a metastatic lesion. Bone and soft tissue lesions may undergo rapid growth in the early weeks after birth but then regress spontaneously during the first few years of life. Although there has been no documentation describing the mechanism of the spontaneous regression of infantile myofibromatosis, Fukasawa et al. postulated that massive apoptosis was responsible ${ }^{8}$.

Microscopically, both the solitary and multicentric forms have similar characteristic appearances with a distinct zoning pattern ${ }^{2,4}$. The peripheral areas of nodular lesions are composed of spindle cells arranged in whorled or interlacing fascicles, giving a leiomyoma-like appearance. These cells demonstrate staining characteristics of both myoblasts and fibroblasts, and frequently contain a large quantity of collagen within the surrounding matrix. In the central areas, a hemangiopericytoma like pattern consisting of cells with less differentiation is usually found. A high mitotic rate greater than 3 mitotic figures per 10 high-power field and infiltration of adjacent adipose tissue and skeletal muscle are not unusual but have no adverse prognostic significance. Myofibroblasts are mesenchymal cells with both features of smooth muscle cells and fibroblasts. Immunostaining for smooth muscle actin and vimentin, with negative staining for desmin can support the myofibroblastic differentiation of infantile myofibromatosis.

The differential diagnosis for infantile myofibromatosis includes other types of fibromatosis, congenital infantile fibrosarcoma, infantile hemangiopericytoma, inflammatory myofibroblastic tumors, fibrohistiocytic tumors with a predominantly fibrous pattern, smooth muscle tumors such as leiomyomas and leiomyosarcomas, neurogenic tumors such as neurofibromas and low-grade malignant peripheral nerve sheath tumors, and nodular fasciitis. 
Myofibromatosis has a number of clinical and morphologic similarities with infantile hemangiopericytoma ${ }^{13}$. These authors proposed that infantile hemangiopericytoma and myofibromatosis represent different stages of maturation of a single entity, a contention supported by others ${ }^{14,15}$.

The pathogenesis of these tumours remains unknown, but its association with estrogen receptors has been postulated 9 , 10. Familial occurrence has been reported in all 3 forms of infantile myofibromatosis. Most cases are sporadic, but autosomal recessive, dominant, and polygenic modes of inheritance have been postulated. Jennings et al $^{9}$ suggested autosomal dominant inheritance with reduced penetration. Venencie et $\mathrm{al}^{12}$ proposed the hypothesis that infantile myofibromatosis is an autosomal recessive condition after the observation of seven siblings. These indicate a need for taking a complete family history in these cases. Cytogenetic and florescence in situ hybridization analyses revealed a pseudodiploid karyotype with an interstitial deletion of the long arm of chromosome 6 del (12q15q), which has been the sole anomaly found ${ }^{7,10}$.

Whether this rare tumor is a true neoplasm or a reactive change is under investigation. Infantile myofibromatosis bears a proliferative feature and some patients even died from the tumor, which support the evidence of neoplastic characters. However, spontaneous regression was observed in some cases suggestive of a self-limiting disorder with reactive nature. Modalities of treatment other than surgery include radiation therapy, local glucocorticosteroid injections and chemotherapy, which have been used with some success when all other modalities failed. Surgery is recommended only if vital structures are affected or if tissue is needed for diagnosis. A few patients with recurrent or non-resectable tumors have been treated with some success with a combination of vincristine, actinomycin-D, and cyclophosphamide with or without radiation. In a study by Jenkins and Cawley ${ }^{11}$, no deaths were reported in patients with the solitary form. In contrast, 29 patients died among 112 multicentric cases. The common causes of death were cardiopulmonary and gastrointestinal complications.

CONCLUSION: Infantile myofibromatosis is a rare mesenchymal tumour. It should be differentiated from other fibrous tissue tumours because of difference in the treatment and prognosis. It should be considered in the evaluation of newborns or young infants presenting with solitary or multiple tumours. Follow up is mandatory because recurrence has been reported in the literature.

\section{REFERENCES:}

1. Maria Letizia Fasola Maccari, Lisandro Manfrin, Maria Laura Galluzo, Andriana Vilma Scrigni. Bol Med Hosp Infant Mex Infantile myofibromatosis: case report and literature review 2011; 68(4):274-278.

2. Wiswell TE, Davis J, Cunningham BE, Solenberger R,Thomas PJ. Infantile myofibromatosis: the most common fibrous tumor of infancy. J Pediatr Surg 1988; 23:314-8.

3. Stout AP. Juvenile fibromatosis. Cancer 1954; 7: 953-78.

4. Chung EB, Enzinger FM. Infantile myofibromatosis. Cancer 1981; 48: 1807-18.

5. Coffin CM, Neilson KA, Ingels S, Frank GR, Dehner LP. Congenital generalized myofibromatosis: A disseminated angiocentric myofibromatosis. Pediatr Pathol Lab Med 1995; 15: 571-87. 
6. Wada H, Akiyama H, Seki H, Ichihara T, Ueno K,Miyawaki T, Koizumi S. Spinal canal involvement in infantile myofibromatosis: Case report and review of the literature. J Pediatr Hematol Oncol 1998; 20: 353-6.

7. Allen PW. The fibromatosis: a clinicopathologic classification based on 140 cases. Review articles, part 2. Am JSurg Pathol 1977; 1:305-21.

8. Fukasawa Y, Ishikura H, Takada A, Yokoyama S, Imamura M, Yoshiki T, Sato H. Massive apoptosis in infantile myofiromatosis: a putative mechanism of tumor regression. Am J Pathol 1994; 144: 480-5.

9. Jennings TA, Duray PH, Collins FS, Sabetta J, Enzinger FM. Infantile myofibromatosis: Evidence for an autosomal-dominant disorder. Am J Surg Pathol 1984; 8: 529-38.

10. Fang-Ying Kuo, MD; Shun-Cheng Huang, MD; Hock-Liew Eng, MD; Jiin-Haur Chuang1, MD; Wei-Jen Chen, MD ,Solitary Infantile Myofibromatosis: Report of Two Cases Chang Gung Med J Vol. 25 No. 6 June 2002

11. Jenkins EA, Cawley MI: Infantile myofibromatosis: A cause of severe bone pain in a neonate. Br J Rhematol1993; 32:849-852.

12. Venencie PY, Bigel P, Desgruelles C, Lortat-Jacob S, Dufier JL, Saurat JH: Infantile myofibromatosis. Report of two cases in one family. Br J Dermatol 1987; 117 :255-259.

13. Mentzel T, Calonje E, Nascimento AG, et al: Infantile hemangiopericytoma versus infantile myofibromatosis. Study of a series suggesting a continuous spectrum of infantile myofibroblastic lesions. Am J Surg Pathol 1994; 18:922.

14. Variend S, Bax NM, van Gorp J: Are infantile myofibromatosis, congenital fibrosarcoma and congenital haemangiopericytoma histogenetically related? Histopathology 1995; 26:57.

15. Dictor M, Elner A, Andersson T, et al: Myofibromatosis-like hemangiopericytoma metastasizing as differentiated vascular smooth-muscle and myosarcoma. Myopericytes as a subset of "myofibroblasts". Am J Surg Pathol 1992; 16:1239

Fig 1 Gross globular soft tissue mass /cut section grey white
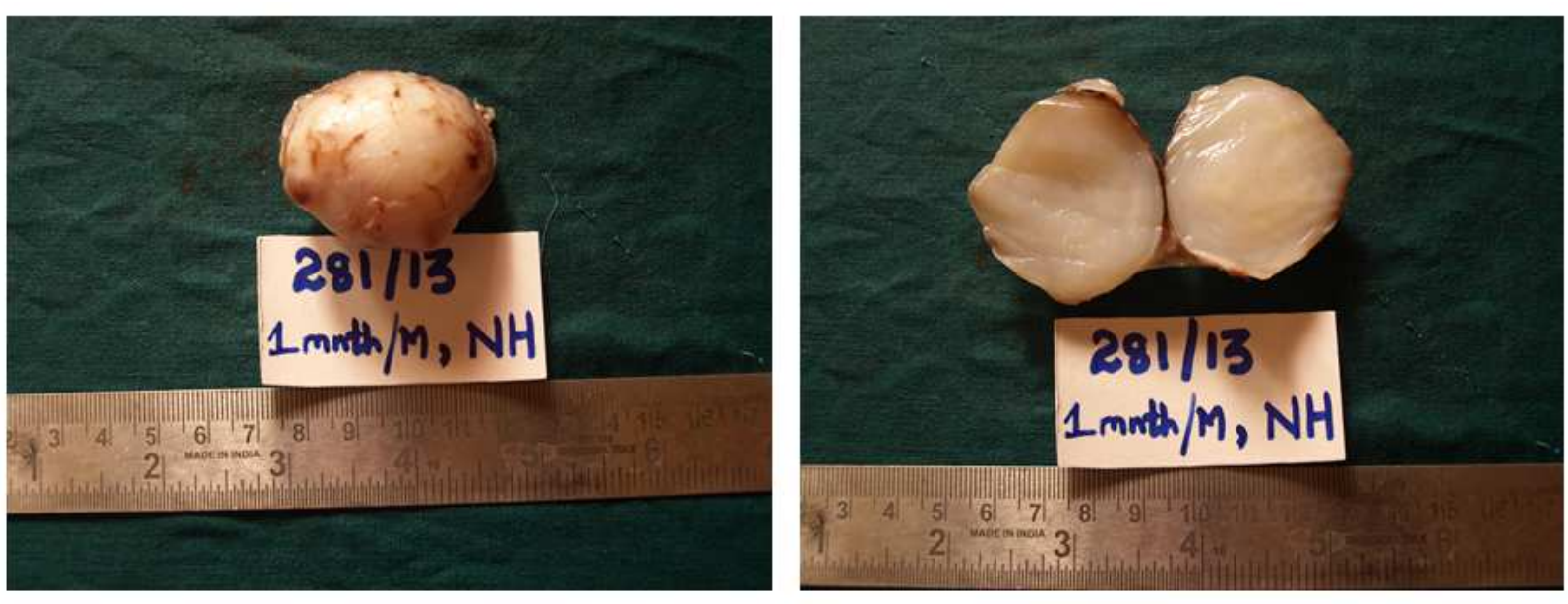

Journal of Evolution of Medical and Dental Sciences/ Volume 2/ Issue 18/ May 6, 2013

Page-3090 


\section{CASE REPORT}

Fig 2 H\&E (10x),spindle shaped cells in bundles and hemangiopericytoma pattern
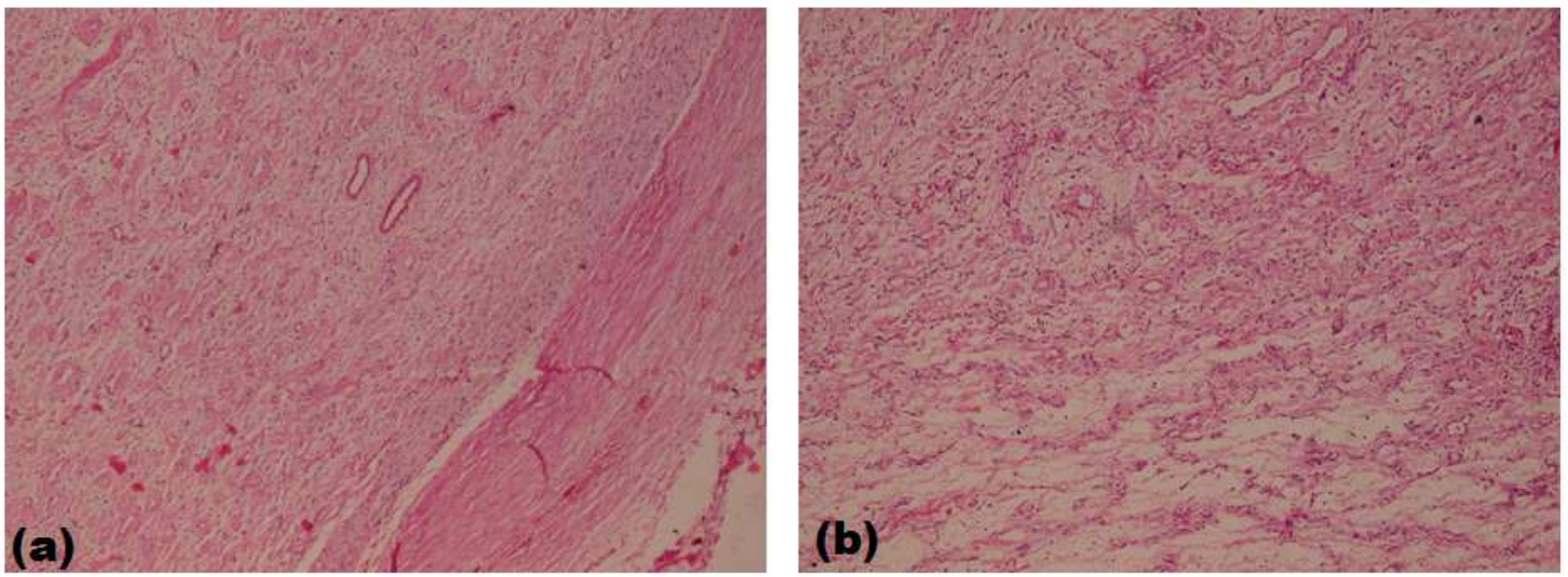

Fig 3 (40x) IHC Vimentin positive

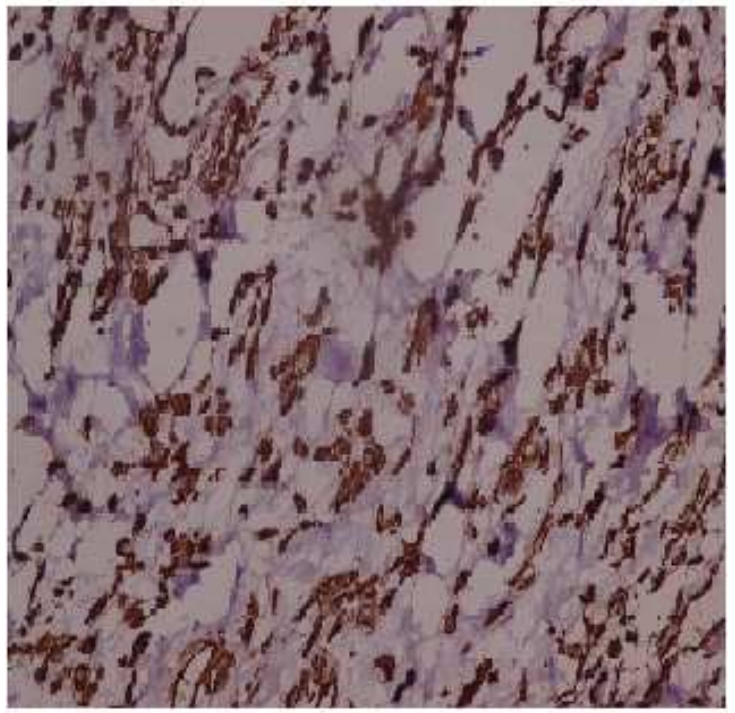

